\title{
Domain structure in biaxial Fe/Cr films induced by lateral fluctuations of the magnetic anisotropy
}

\author{
A. A. Rzhevsky \\ Institut für Festkörperforschung IFF-9 “Elektronische Eigenschaften,” Forschungszentrum Jülich GmbH, D-52425 Jülich, Germany \\ and Ioffe Physical Technical Institute, Russian Academy of Sciences, 194021 St. Petersburg, Russia \\ B. B. Krichevtsov \\ Ioffe Physical Technical Institute, Russian Academy of Sciences, 194021 St. Petersburg, Russia \\ D. E. Bürgler and C. M. Schneider \\ Institut für Festkörperforschung IFF-9 “Elektronische Eigenschaften,” Forschungszentrum Jülich GmbH, D-52425 Jülich, Germany
}

(Received 3 November 2007; revised manuscript received 9 April 2008; published 30 May 2008)

\begin{abstract}
The magnetic microstructure of single-crystalline Fe(001) thin films has been studied by magnetic second harmonic generation and conventional magneto-optical Kerr effect methods. The layers were grown on $\mathrm{GaAs} / \mathrm{Fe} / \mathrm{Ag}(100)$ substrates, capped by a Cr overlayer, and displayed a fourfold in-plane magnetic anisotropy. We observe the formation of a multidomain structure at magnetic fields $|H| \leq 0.5 \mathrm{kOe}$, when the field is applied in a narrow range of azimuthal angles close to the hard axis direction $\left(|\Delta \xi|<1^{\circ}\right)$. The domains are characterized by the same longitudinal magnetization component $M_{x}$ and transverse components $M_{y}$ of opposite signs. We developed an approach to extract the change of the relative contributions of domains with different magnetization directions during magnetization reversal from the experimental data. The formation of a domain structure can be explained by taking into account the lateral fluctuations of the magnetic anisotropy energy caused by defects and stress at the interfaces and the role of the biquadratic exchange coupling between interfacial $\mathrm{Fe}$ and $\mathrm{Cr}$ magnetic moments.
\end{abstract}

DOI: 10.1103/PhysRevB.77.174432

PACS number(s): 42.65.Ky, 75.60.-d, 75.70.Cn, 78.20.Ls

\section{INTRODUCTION}

The magnetic properties of the epitaxial $\mathrm{Fe} / \mathrm{Cr}$ film system as well as the behavior of exchange-coupled $\mathrm{Fe} / \mathrm{Cr} / \mathrm{Fe}$ structures based on it have been the subject of intensive studies for a long time. This material combination was the first to exhibit the giant magnetoresistance (GMR) effect, ${ }^{1,2}$ a discovery that started off the field of spintronics. GMR is still of major interest for applications and devices in spintronics, ${ }^{3,4}$ e.g., in read heads, magnetic field sensors, magnetic access memories, etc. Since the magnetic state of $\mathrm{Fe} / \mathrm{Cr}$ and $\mathrm{Fe} / \mathrm{Cr} / \mathrm{Fe}$ structures can be changed by a relatively weak magnetic field, these layer stacks may be considered as model systems for studying the many facets of thin film magnetism. ${ }^{5}$

The magnetic properties of $\mathrm{Fe} / \mathrm{Cr}$ and $\mathrm{Fe} / \mathrm{Cr} / \mathrm{Fe}$ have been studied by a variety of methods such as Brillouin light scattering (BLS), ferromagnetic resonance (FMR), neutron scattering, or the magneto-optical Kerr effect (MOKE), and a compilation of the major results can be found in several review articles. ${ }^{5-8}$ Recently, the transformation between different magnetic states in exchange-coupled structures $\mathrm{Fe} / \mathrm{Cr} / \mathrm{Fe}$ has been probed by the method of magnetization-induced second harmonic generation (MSHG). ${ }^{9}$ We have shown that MSHG opens a pathway to explore peculiarities of the magnetic state and its field-induced evolution in thin films, which have not been observed previously by using conventional methods, e.g., MOKE or BLS. In particular, when an inplane magnetic field was applied close to the direction of the hard axis in an $\mathrm{Fe} / \mathrm{Cr} / \mathrm{Fe}$ structure-displaying a ferrimagnetic alignment at $H=0$-we observed an additional mag- netic state transformation by means of MSHG. For the correct interpretation of the experimental results obtained by the different methods (FMR, BLS, MSHG) as well as for the practical application of $\mathrm{Fe} / \mathrm{Cr}$ structures, a detailed knowledge of their magnetic properties, in particular, the domain structure at different values and orientations of the magnetic field, is needed.

In this work, we employ the MSHG approach to probe and analyze the domain structure of anisotropic $\mathrm{Fe} / \mathrm{Cr}$ films. The MSHG response in the case of a multidomain state has been studied both experimentally and theoretically. With the help of MSHG the domain images of antiferromagnetic $\mathrm{Cr}_{2} \mathrm{O}_{3},{ }^{10}$ hexagonal rare-earth manganites $R \mathrm{MnO}_{3},{ }^{11,12}$ as well as magnetic garnet films ${ }^{13,14}$ have been obtained. The MSHG features in the case of a multidomain structure in contrast to a single-domain case were theoretically considered in Refs. 15-17. In particular, a complementary mechanism associated with a second harmonic generation signal arising from domain walls was proposed in Ref. 15. This particular mechanism is based on the spatial magnetization gradients $\nabla \mathbf{M}$ in the domain wall. For the situation of magnetic stripe domains it was predicted that the angular profile of the SHG intensity comprises a diffraction pattern of SHG beams characterized by diffraction orders $m$. The detailed theory of SHG and MSHG from magnetic multilayers including the case of a multidomain structure and calculations of the angular profiles of these phenomena based on the electric point-dipole radiation theory were recently presented in Ref. 17.

The appearance of a domain structure in systems with in-plane uniaxial magnetic anisotropy when a magnetic field 
is applied along the hard axis, ${ }^{18,19}$ as well as in systems with out-of-plane magnetic anisotropy when a magnetic field is applied perpendicular to the easy axis, is well known. ${ }^{20}$ The formation of this structure is associated with fluctuations of the magnetic anisotropy appearing for different reasons related to the methods of preparation, the homogeneity, uniformity, morphological structure of the film, and so on. In Ref. 20 the formation of a multidomain structure during the magnetization reversal process initiated by an in-plane magnetic field in polycrystalline $\mathrm{Au} / \mathrm{Co} / \mathrm{Au}$ structures having strong perpendicular magnetic anisotropy has been studied. The observed phenomenon was associated with the existence of nanosized uncoupled atomic terraces with different local anisotropies. Nevertheless, to the best of our knowledge in systems with biaxial magnetic anisotropy and particularly in epitaxial, monocrystalline $\mathrm{Fe} / \mathrm{Cr}(100)$ films grown by molecular beam epitaxy (MBE), the existence of a nonuniform magnetic state in a wide range of magnetic field including $H=0$ has not yet been reported.

The domain structures in poly- and monocrystalline $\mathrm{Fe} / \mathrm{Cr}$ and $\mathrm{Cr} / \mathrm{Fe}$ films have been studied by different methods. In the work of Fischer et al. in Ref. 21, by means of magnetic transmission $\mathrm{x}$-ray microscopy, images of in-plane magnetic domains in $\mathrm{Cr}(3 \mathrm{~nm}) / \mathrm{Fe}(50 \mathrm{~nm}) / \mathrm{Cr}(6 \mathrm{~nm})$ films grown by thermal evaporation on $\mathrm{Si}_{3} \mathrm{~N}_{4}$ substrates were obtained with a magnetic field applied in the film plane. In polycrystalline films with a weak uniaxial in-plane magnetic anisotropy a typical ripple structure with preferred domain orientations was observed. The latter were attributed to small magnetic fields being present during the evaporation process. The domain structure of thin $(2 \mathrm{~nm}) \mathrm{Fe}$ monocrystalline films grown by MBE on bulk $\mathrm{Cr}(100)$ using secondary electron microscopy with polarization analysis was studied in Ref. 22. At temperatures above $T_{N}$ of bulk $\mathrm{Cr}$, the ultrathin films are left in a monodomain state with the magnetization along the easy axis after application and removal of the external magnetic field. However, at temperatures lower than $T_{N}$, a transition to a multidomain state takes place, with the magnetization still lying in the film plane, but the magnetization vector rotated by $90^{\circ}$ in some areas. The reason for the appearance of the multidomain state with the orthogonal direction of $\mathbf{M}$ at $T$ $<T_{N}$ is associated with the presence of an atomic scale roughness at the interface, which can result in perpendicular coupling of $\mathrm{Fe}$ and $\mathrm{Cr}$ interfacial magnetic moments. Note that such an unusual behavior was observed in Fe films with a thickness of less than $5 \mathrm{~nm}$. In Ref. 23 the domain structure of epitaxial $\mathrm{Fe} / \mathrm{Cr}(100)$ thin films was studied by means of element-sensitive photoemission spectroscopy based on magnetodichroic effects in photoinduced Auger electron emission. The images of $180^{\circ}$ domains in $\mathrm{Cr}$ films and $\mathrm{Fe}$ substrates were separately obtained with spatial resolution of $10 \mu \mathrm{m}$, and regions of bilinear and biquadratic exchange coupling in a $\mathrm{Fe} / \mathrm{Cr} / \mathrm{Co}$ sandwich were identified.

In this contribution we show that in monocrystalline biaxially anisotropic $\mathrm{Fe} / \mathrm{Cr}$ films of $10-50 \mathrm{~nm}$ thickness a peculiar magnetic multidomain structure with equal components of the longitudinal magnetization $M_{x}$ and transverse components $M_{y}$ of different sign can be realized at $H=0$, after applying a saturating magnetic field in a narrow angle range slightly deviating from the hard axis direction. The
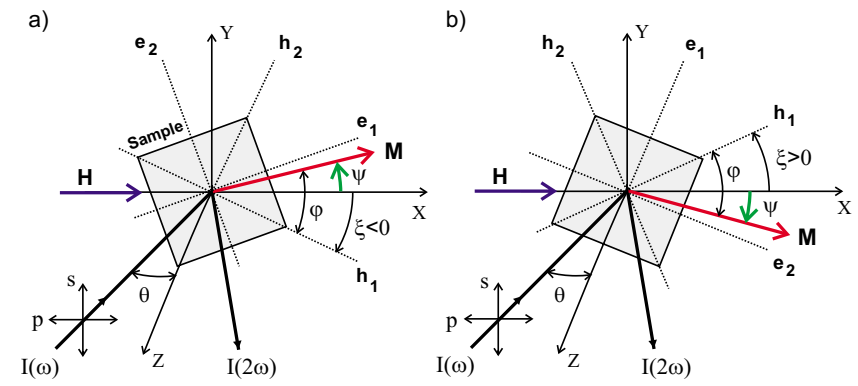

FIG. 1. (Color online) Geometry of the MSHG experiment and orientation of magnetization $\mathbf{M}$ at negative (a) and positive (b) azimuth $(\xi)$ of the hard axis $\mathbf{h}_{1}$ in a fourfold anisotropic system. Here the $\left(\mathbf{h}_{1}, \mathbf{h}_{2}\right)$ hard and $\left(\mathbf{e}_{1}, \mathbf{e}_{2}\right)$ easy axes correspond to [110]- and [100]-type crystallographic directions in the film plane, respectively. $X, Y, Z$ is the laboratory coordinate system; $\varphi$ the angle between the magnetization and hard axis $\mathbf{h}_{1} ; \psi$ is the azimuthal angle of the magnetization, with the arrows showing the direction of the magnetization rotation as the magnetic field $H$ decreases; $\theta$ is the angle of incidence.

analysis of the MSHG experimental data obtained allows us to define the change of the relative contributions of domains with different $M_{y}$ components during the magnetization reversal process.

\section{EXPERIMENTAL ASPECTS}

The Fe films (thickness $d=10-50 \mathrm{~nm}$ ) were grown by molecular beam epitaxy onto GaAs(001) substrates, with an $\operatorname{Ag}(150 \mathrm{~nm}) / \mathrm{Fe}(1 \mathrm{~nm})$ buffer layer being deposited prior to the $\mathrm{Fe}$ film growth in order to provide better epitaxy. ${ }^{24}$ The structure was covered by a $\operatorname{Cr}(2 \mathrm{~nm})$ protective cap layer. The quality of the films has been monitored in situ by reflection high-energy electron diffraction during the growth process. After deposition the sample was removed from the chamber and fixed onto a sample holder allowing a $360^{\circ}$ rotation around the surface normal. All measurements were performed at room temperature $(T=294 \mathrm{~K})$.

The geometry of the MSHG experiment is schematically shown in Fig. 1. A magnetic field up to $3 \mathrm{kOe}$ was applied parallel to the sample surface in the plane of the incident light (longitudinal geometry). The MSHG field dependencies were measured in an applied magnetic field by sweeping in the range $0 \Rightarrow 3 \mathrm{kOe} \Rightarrow-3 \mathrm{kOe} \Rightarrow 3 \mathrm{kOe}$. The exciting light pulses at $\lambda=800 \mathrm{~nm}\left(E_{\mathrm{ph}}=\hbar \omega=1.55 \mathrm{eV}\right)$ with a duration of $150 \mathrm{fs}$ were generated by a Ti:sapphire regenerative amplifier with $1 \mathrm{kHz}$ repetition rate and $15 \mu \mathrm{J}$ pulse power. The pulses were focused into a spot of $1 \mathrm{~mm}$ diameter on the sample surface. The SHG was measured in reflection at $\lambda$ $=400 \mathrm{~nm}\left(E_{\mathrm{ph}}=2 \hbar \omega=3.1 \mathrm{eV}\right)$. The angle of incidence was $\theta \approx 5^{\circ}$. The polarization of the fundamental and frequencydoubled light was chosen by a proper orientation of polarizer and analyzer, allowing one to investigate the second harmonic generation (SHG) signal in four different polarization combinations $(p p, p s, s s, s p)$. The fundamental light at $\lambda$ $=800 \mathrm{~nm}$ was rejected by placing a blue filter (BG-39) into the reflected beam. The SHG signal was recorded using a photomultiplier and photon counting technique. The count- 
ing time of each experimental point was 10-20 s. While the polarizer was rotated, the intensity of the excited light was kept constant (with an uncertainty of less than 5\%) by means of a quarter-wave plate placed just before the polarizer. The sample was mounted on a motorized rotational stage providing azimuthal rotation around the surface normal with high precision $\left(\sim 0.001^{\circ}\right)$.

For comparison purposes, together with the MSHG also the conventional longitudinal magneto-optical Kerr effect has been studied in the $\mathrm{Fe} / \mathrm{Cr}$ films using a $\mathrm{cw}$ diode laser at $\lambda=670 \mathrm{~nm}$ for $s$ polarization of the fundamental beam. The incidence angle in these measurements was kept at about $35^{\circ}$. A high sensitivity of the MOKE measurements was obtained by using a differential photodetector and lock-in technique.

\section{EXPERIMENTAL RESULTS AND DISCUSSION}

\section{A. MSHG approach}

In order to extract details of the interfacial magnetization behavior in $\mathrm{Fe} / \mathrm{Cr}$ structures we have to describe the field dependencies of the MSHG response for different polarization combinations of the light. For this purpose we follow the formalism used in Ref. 9. The formalism is based on phenomenological expressions of the nonlinear response from the single surface or interface of $C_{4 v}$ symmetry. ${ }^{25-30}$ As shown in Ref. 9, these expressions can also be used to describe the nonlinear response of the system with several interfaces-provided that all of them have the same $C_{4 v}$ symmetry. In that case, the description of the system with more than one interface can be conducted in terms of effective nonlinear optical susceptibilities $\left(\chi^{\mathrm{eff}}\right)$. The expressions for the SHG intensity at small incidence angles for $p p$ and $s s$ polarization combinations have the forms

$$
\begin{gathered}
I_{2 \omega}=A\left|\chi_{x x x}^{\text {eff }} M_{y}+\alpha_{n} \theta\right|^{2}=A\left(\left|\chi_{x x x}^{\text {eff }}\right|^{2} M_{y}^{2}+\left|\alpha_{n} \theta\right|^{2}\right. \\
\left.+2\left|\chi_{x x x}^{\text {eff }} \| \alpha_{n}\right| \theta M_{y} \cos \Delta\right) \text { for } p p, \\
I_{2 \omega}=A\left|\chi_{y y y}^{\text {eff }}\right|^{2} M_{x}^{2} \text { for } s s,
\end{gathered}
$$

where $A$ is a parameter depending on the intensity of the fundamental light; $\chi_{i j k}^{\text {eff }}$ are the effective nonlinear optical susceptibilities; $\theta$ is the incidence angle; $\alpha_{n}=\left(2 \chi_{x z x}^{n} / n+N \chi_{z x x}^{n}\right)$, where $\chi_{i j k}^{m}$ and $\chi_{i j k}^{n}$ are the linear-in-M and nonmagnetic nonlinear optical susceptibilities, respectively; $N$ and $n$ are refractive indices at $2 \omega$ and $\omega$; and $\Delta$ defines the phase shift, since the parameters $\chi^{\text {eff }}$ and $\alpha_{n}$ are complex numbers. As follows from the expressions (1) and (2) the nonmagnetic nonlinear susceptibility in the $p p$ polarization combination is proportional to the incidence angle $\theta$, while the contribution of the magnetic nonlinear susceptibility is defined by the $y$ component of the film magnetization. In contrast, for the ss polarization combination, the nonmagnetic nonlinear susceptibility component is absent, but the contribution of the magnetic one is defined by the $x$ component of the magnetization. The linear-in-magnetization contribution in the SHG signal might appear in $p p$ polarization combinations as a result of the interference of magnetic and nonmagnetic contributions.
In the $s s$ polarization combination, the SHG signal is defined by quadratic-in-magnetization terms only.

As shown in Ref. 9, the expressions (1) and (2) can be used to describe the experimental field dependencies of the SHG response in Fe/Cr films, provided that the changes of the $M_{x}$ and $M_{y}$ components depending on the magnitude and direction of the applied magnetic field $\mathbf{H}$ are known. To calculate the $M_{x, y}(H)$ field dependencies, the expression for the magnetic energy density $\varepsilon_{m}$ including the Zeeman energy and the energy of the biaxial anisotropy and assuming an in-plane orientation of the magnetization $\left(M_{z}=0\right)$ should be used:

$$
\varepsilon_{m}=-H M_{s} \cos \psi+\frac{K_{1}}{4} \cos ^{2}(2 \varphi),
$$

where $M_{s}$ is the saturation magnetization, $K_{1}$ is the fourfold in-plane magnetic anisotropy constant, $\psi$ is the angle between the magnetization $\mathbf{M}$ and magnetic field $\mathbf{H}, \varphi$ is the angle between $\mathbf{M}$ and the hard axis $\mathbf{h}_{1}$ (see Fig. 1). The magnetization value $M=1.71 \mathrm{kG}$ of bulk Fe and the expression $2 K_{1} / M_{s}=[0.55-2.5 / d(M L)] \mathrm{kOe}$ of Ref. 6 to define the anisotropy constant have been used in the calculations of $\varepsilon_{m}$ The minimization of Eq. (3) gives the possibility of defining the orientation of the magnetization $\mathbf{M}$ in the film plane depending on the direction and magnitude of the applied magnetic field $\mathbf{H}$. The parameters $\left|\chi^{\text {eff }}\right|,\left|\alpha_{n}\right|$ and the phase shift $\Delta$ are defined from the experimental values $I_{2 \omega}(H \rightarrow+0), I_{2 \omega}(H \rightarrow-0)$, and $I_{2 \omega}\left(H_{s}\right)$ for corresponding polarization combinations $(p p, s s)$.

It should be noted that the MSHG signal reflects the magnetization behavior only close to the interfaces within a depth of one or two atomic layers. In contrast to that, the conventional MOKE signal is mainly related to the volume magnetization. However, previous investigations of MSHG and $\mathrm{MOKE}$ in $\mathrm{Fe} / \mathrm{Cr} / \mathrm{Fe}$ structures showed that the volume and interfacial magnetization behave in a similar way. ${ }^{9}$ In particular, the critical fields corresponding to jumplike transformations of the magnetic structure are the same for both MOKE and MSHG.

\section{B. Domain structure and estimation of relative domain volumes}

In Figs. 2(a)-2(d) the field dependencies of $I_{2 \omega}(H)$ for $p p$ and $s s$ polarization combinations in $\mathrm{Fe}(10 \mathrm{~nm}) / \mathrm{Cr}$ films at a deviation of the hard axis $\mathbf{h}_{1}$ by $\xi= \pm 2^{\circ}$ from the direction of the magnetic field $\mathbf{H}$ are shown. The solid lines correspond to calculations on the basis of Eqs. (1) and (2). In the range of positive fields $(H>0)$, the calculated curves correspond to a coherent rotation of the magnetization toward the nearest easy axis as the field decreases, i.e., $\mathbf{e}_{1}$ for $\xi=-2^{\circ}$ and $\mathbf{e}_{2}$ for $\xi=+2^{\circ}$ (see Fig. 1). The jumps of $I_{2 \omega}$ close to $H \approx 0$ are due to a change of sign of the $M_{y}$ component. In the range of negative fields $(H<0)$, the theoretical curves correspond to a magnetization rotation from the easy axis toward the direction of $\mathbf{H}$ as the field amplitude increases. For the $p p$ polarization combination the field dependencies $I_{2 \omega}(H)$ measured at a deviation of the magnetic field from the hard axis by the same angle $\xi= \pm 2^{\circ}$ display a mirror symmetry rela- 


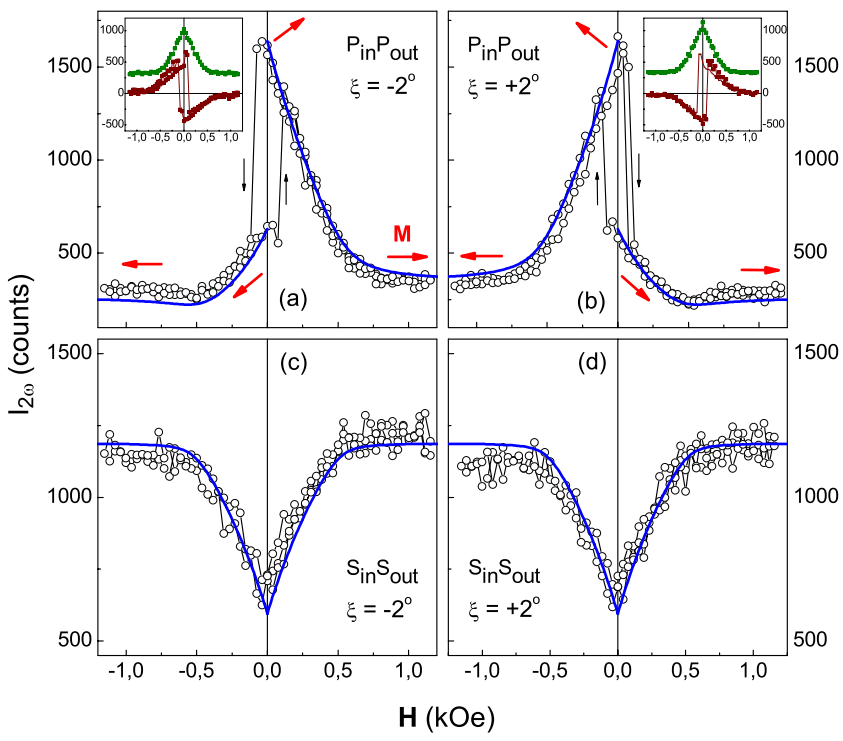

FIG. 2. (Color online) SHG field dependencies in $\mathrm{Fe}(10 \mathrm{~nm}) /$ $\mathrm{Cr}(2 \mathrm{~nm})$ films in $p p(\mathrm{a}),(\mathrm{b})$ and $s s$ (c),(d) polarization combinations with the angle between magnetic field $(\mathbf{H})$ and the hard axis $\mathbf{h}_{1} \xi$ $= \pm 2^{\circ}$. Insets: the odd $\left(\sim M_{y}\right)$ and even $\left(\sim M_{y}^{2}\right)$ contributions extracted from the measured SHG signal in the $p p$ polarization combination. The lines are calculations performed with use of Eqs. (1) and (2) and parameter values $A\left|\alpha_{n} \theta\right|^{2}=300$ (counts), $A\left|\chi_{x x x}^{\text {eff }}\right|^{2} M^{2}$ $=1670 \quad$ (counts), $2 A\left|\chi_{x x x}^{\text {eff }} \| \alpha_{n}\right| M \theta \cos \Delta=765 \quad$ (counts), and $A\left|\chi_{y y y}^{\text {eff }}\right|^{2} M^{2}=1190$ (counts). The thick arrows schematically show the magnetization orientation in the $X Y$ plane.

tive to the ordinate axis $(H=0)$ [Figs. 2(a) and 2(b)]. The strong increase of $I_{2 \omega}$ in the $p p$ polarization experiment during a decrease of the magnetic field amplitude $(|H| \rightarrow 0)$ from positive for $\xi=-2^{\circ}$ and negative for $\xi=+2^{\circ}$ values is caused by the fact that in both cases the positive $M_{y}$ component increases. Therefore, the increase of $I_{2 \omega}$ is due to the last interference term in Eq. (1). In other cases at decreasing magnetic field the $M_{y}$ component with negative sign increases. Consequently, the interference term in Eq. (1) leads to a weakening of the SHG signal. For the ss polarization combination the field dependencies $I_{2 \omega}(H)$ are symmetrical with respect to the magnetic field [Figs. 2(c) and 2(d)]. In the ss polarization geometry, the SHG signal is proportional to $M_{x}^{2}$; therefore it does not suffer from a discontinuity when $M_{x}$ changes sign near $H=0$. In this case the $I_{2 \omega}$ dependence is symmetric in the magnetic field and weakly depends on the angle between magnetic field and hard axis.

As is seen in Fig. 2 at a deviation of the magnetic field from the hard axis by about $2^{\circ}$ the multidomain structure forms only in the discontinuity area after the magnetic field changes sign, but at $H=0$ the film still remains in the monodomain state. For this case the MSHG loops for $p p$ polarization can be decomposed into parts even and odd in the magnetic field. According to Eq. (1), the even part is related to the constant term $\left|\alpha_{n} \theta\right|^{2}$ and the term proportional to $M_{y}^{2}$. The odd part of the loop is determined only by the $M_{y}$ component. The results of the decomposition are shown in the insets of Figs. 2(a) and 2(b). Note that this procedure is valid only for cases when during the magnetization process the film remains mainly in the monodomain state. In the case of

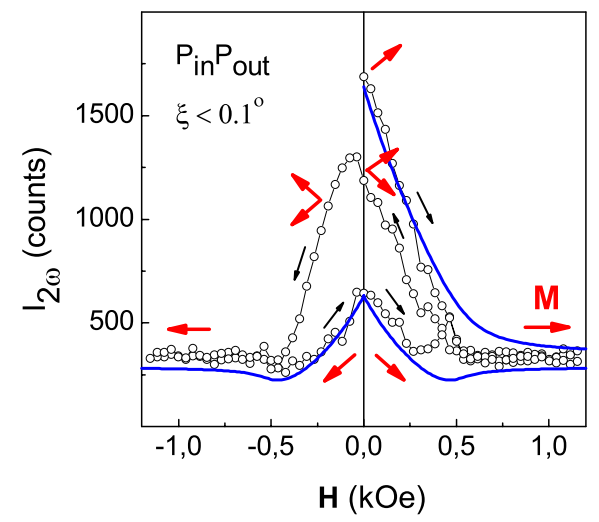

FIG. 3. (Color online) SHG field dependence $\mathrm{Fe}(10 \mathrm{~nm}) / \mathrm{Cr}(2$ $\mathrm{nm})$ films in $p p$ polarization combination with the angle between magnetic field $(\mathbf{H})$ and the hard axis $\left(\mathbf{h}_{1}\right) \xi<0.1^{\circ}$. The lines are calculations performed with use of Eq. (1) with the same parameter values as in Fig. 2. The thick arrows schematically show the magnetization state and orientation of the magnetization in domains.

multidomain state a more elaborate analysis is needed.

In Fig. 3 for the same film the $I_{2 \omega}(H)$ dependence for the $p p$ polarization combination and $\xi<0.1^{\circ}$ is presented. The dependence is very different from those shown in Figs. 2(a) and 2(b). In analogy to the dependence in Fig. 2(a), it starts from the monodomain state being conserved after the measurement cycle at $\xi=-2^{\circ}$. As the field increases, it achieves the saturation value and the magnetization aligns along the field. However, with a subsequent decrease of the field to $H=0$, the initial value of $I_{2 \omega}(H)$ is not reached. This means that the magnitude of $M_{y}$ in this case is essentially smaller than the initial value corresponding to the monodomain state. After the field changes sign, $I_{2 \omega}(H)$ approaches the saturation value at negative field $-H_{s}$. In the reversed field direction (from $-H_{s}$ to 0$) I_{2 \omega}(H)$ changes, corresponding to a coherent rotation of the magnetization in the monodomain state. In the area of $\left(0,+H_{s}\right)$ also a coherent rotation of the magnetization takes place. Note that, when the field changes sign in the $\left(-H_{s}, H_{s}\right)$ area, the sign of the $M_{y}$ component does not change. The effective decrease of the $M_{y}$ component as the field decreases from positive values evidences the presence of a multidomain state in the film involving opposite signs of the $M_{y}$ component in neighboring domains.

In Figs. 4(a)-4(f) the SHG field dependencies in an $\mathrm{Fe}(49$ $\mathrm{nm}) / \mathrm{Cr}(2 \mathrm{~nm})$ system at different azimuthal angles of the magnetic field close to the hard axis are shown. The solid lines show the results of calculations for the case of coherent rotation of the magnetization to different easy axes. Thus, if a coherent rotation of $\mathbf{M}$ toward one of the easy axes in the film takes place, we expect the field dependence to agree with the calculated curves. However, if the system develops a multidomain structure, the experimental dependencies will fall inside the area formed by the calculated curves. In fact, a coherent rotation of the magnetization is observed in Figs. 4(a) and 4(b), while the existence of a multidomain structure in a wide range of field values (including zero magnetic field) is clearly seen in Figs. 4(c)-4(f). Note that the experimental dependence presented in Fig. 4(e) is nearly symmetrical—analogously to that observed in the $\mathrm{Fe}(10 \mathrm{~nm})$ 


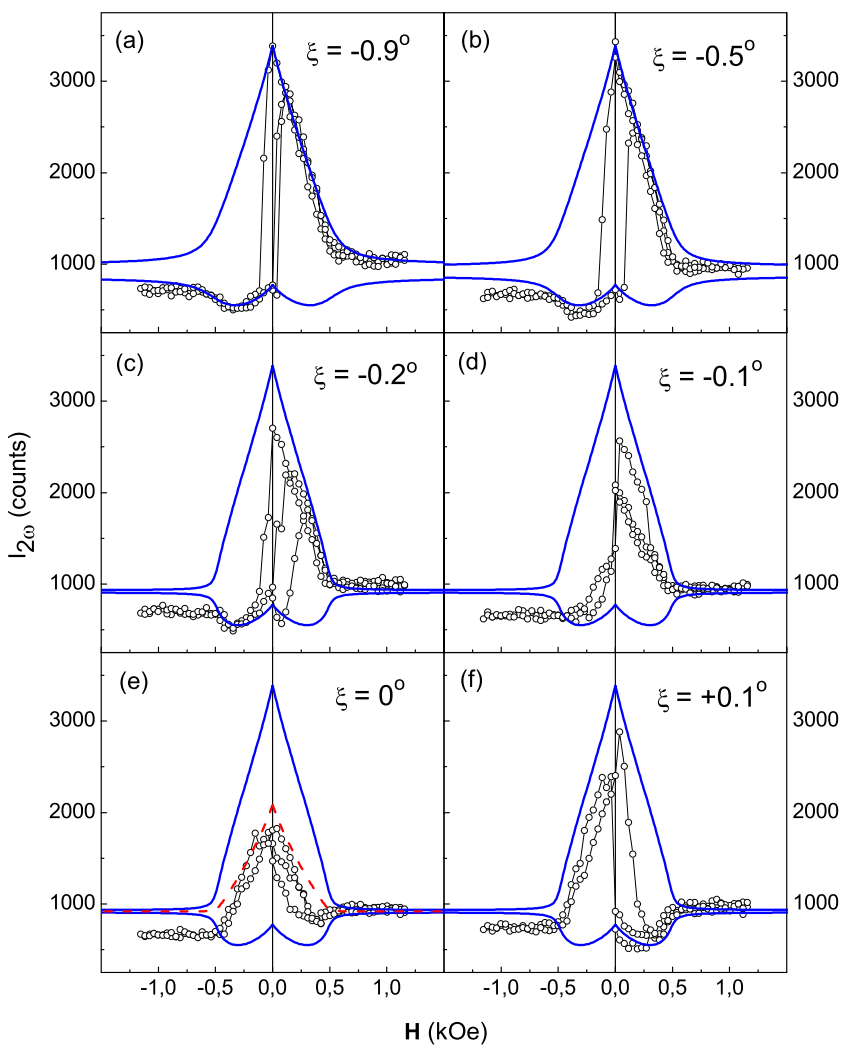

FIG. 4. (Color online) SHG field dependencies in Fe(49 nm)/ $\mathrm{Cr}(2 \mathrm{~nm})$ films in $p p$ polarization combination for different azimuths $(\xi)$ of the hard axis. The lines are the calculations of the magnetization coherent rotation to the different easy axes performed with use of Eqs. (1) and (2) with the following parameter values: $A\left|\alpha_{n} \theta\right|^{2}=924$ (counts), $A\left|\chi_{x x x}^{\text {eff }}\right| M^{2}=2340$ (counts), and $2 A\left|\chi_{x x x}^{\text {eff }} \| \alpha_{n}\right| M \cos \Delta=937$ (counts).

film for ss polarization [see Figs. 2(c) and 2(d)]. The latter, according to Eq. (2), is defined only by terms quadratic in magnetization. The $I_{2 \omega}(H)$ dependence in the $p p$ polarization combination is defined by terms both quadratic and linear in $M_{y}$ [see Eq. (1)]. If in the exposed area equal volumes of domains with positive and negative components of $M_{y}$ exist, then the linear contributions to the SHG signal from these areas compensate each other and $I_{2 \omega}(H)$ will be defined only by the terms quadratic in magnetization. The contributions of these terms arising from different domain types will be equal, i.e., the field dependence will be symmetric. The realization of this scenario is shown in Fig. 4(e) by a dashed line. It is natural to consider this film orientation as corresponding to $\xi=0$, i.e., $(\mathbf{h} \| \mathbf{H})$. At larger values of the field $H>2 \mathrm{kOe}$ the magnetization orientation is already so close to the direction of the magnetic field that the difference in $M_{y}$ components is small. Nevertheless, one may assert that, as the amplitude of the magnetic field decreases from $H_{s}$ down to zero, the magnetization in different areas of the film rotates to competing easy axes. This happens when a magnetic field decreases from positive as well as from negative fields. Basing on the calculated curves $I_{2 \omega}(H)$ shown in Fig. 4 by solid lines, one may calculate the field dependencies of the relative volumes of domains $W_{1}(H)$ and $W_{2}(H)$ with opposite orientations of the $M_{y}$ component. In the $p p$ polarization combi-

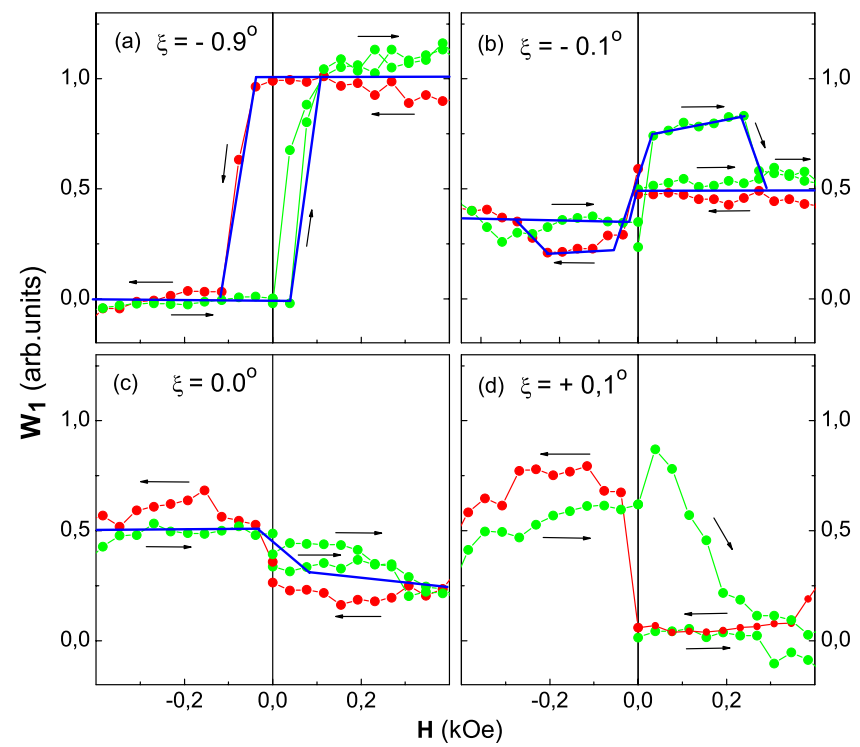

FIG. 5. (Color online) Field dependencies of the relative domain volume $\left(W_{1}\right)$ characterized by positive $M_{y}$ component at different azimuths $(\xi)$ calculated with use of Eqs. (4) and (5). The same experimental data as shown in Fig. 4 are used.

nation the dependence $I_{2 \omega}(H)$ in the multidomain state can be written in the form

$$
I_{2 \omega}^{\mathrm{multi}}(H)=W_{1}(H) I_{2 \omega}^{\mathrm{calc}}\left(+M_{y}(H)\right)+W_{2}(H) I_{2 \omega}^{\mathrm{calc}}\left(-M_{y}(H)\right),
$$

$$
W_{1}(H)+W_{2}(H)=1,
$$

where $I_{2 \omega}^{\text {calc }}\left( \pm M_{y}(H)\right)$ are the values of the SHG intensity calculated on the basis of Eq. (1). The solution of the system of Eqs. (4) and (5) allows us to calculate $W_{1}(H)$ and $W_{2}(H)$. As we can see in Fig. 4, the experimentally observed magnitude of $I_{2 \omega}$ for positive and negative saturating field is slightly different. This small difference, which is absent in the theoretical curves, can be attributed to a small deflection of magnetic field from the incidence plane that results in small contributions of $M_{x}$ to $I_{2 \omega}$. This contribution has been taken into account in the calculations and the results are shown in Fig. 5.

At a deviation of the magnetic field $\mathbf{H}$ from the hard axis $\left(\xi=-0.9^{\circ}\right)$ the $W_{1}(H)$ dependence represents a hysteresis loop of rectangular shape. In fields $|H|>0.12 \mathrm{kOe}$ in the exposed area the monodomain states characterized by $M_{y}$ components of opposite sign are realized. At $\xi=-0.1^{\circ}$ the rectangular hysteresis is absent and a monodomain state is not established. Instead a quite complicated transformation of the domain structure accompanied by jumps of $W_{1}$ is observed. In particular, in the region $H=0.3 \mathrm{kOe}$ [Fig. 5(b)] a sharp decrease of $W_{1}$ in increasing field is observed. In a sweep of the magnetic field in the reversed direction, an increase of $W_{1}$ is observed in the same field regime. At $\xi$ $=0^{\circ}$ the jumps are absent and the $W_{1}$ average values in the positive and negative field areas differ somewhat. This indicates that the volumes of different domains are still changing with the field even in this case. At $\xi=+0.1^{\circ}$ the rectangular 


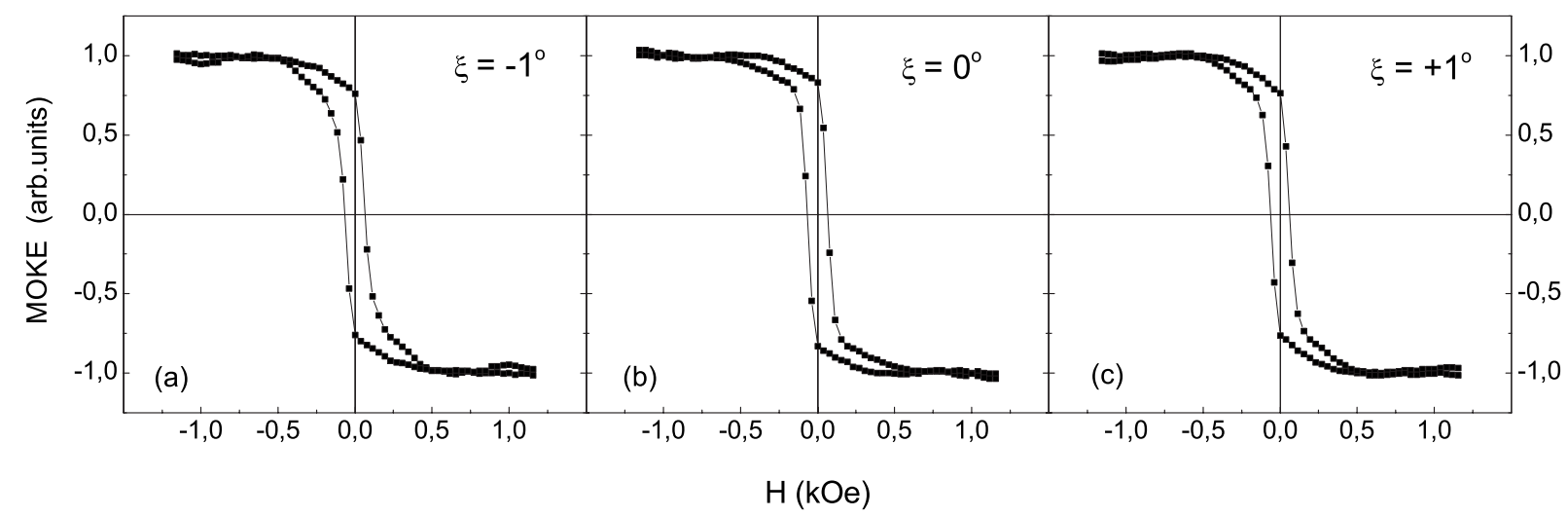

FIG. 6. Longitudinal MOKE in the $\mathrm{Fe}(49 \mathrm{~nm}) / \mathrm{Cr}(2 \mathrm{~nm})$ film at different angles $(\xi)$ between the directions of the magnetic field and hard axis.

loop starts to appear; however, in the negative field range the monodomain state is not yet achieved. Such an interpretation assumes that the lateral domain dimensions with positive and negative $M_{y}$ components may be comparable with the probed area. As follows from the theoretical arguments discussed in Ref. 17 , in the case of a stripe domain structure with a period comparable to or exceeding the light wavelength, diffraction peaks should be observable in the angular distribution of the SHG signal. In addition, in the specular reflection geometry, the nonlinear susceptibilities odd in the magnetic field should not appear. The fact that in our measurements sizable linear-in-M susceptibilities are observed means that diffraction effects do not play a significant role. This also suggests that the domain dimensions are much larger than the wavelength of the light. The magnetic contrast of the SHG signal from different domain areas is $60 \%$ at $H \sim 0.1 \mathrm{kOe}$. This relatively large value should enable a visualization of these domain structures by means of a charge-coupled device camera.

In Fig. 6 the field dependencies of the longitudinal magneto-optical Kerr effect at different $\xi$ are shown. It should be noted that the polarization plane rotation observed in the experiment at a magnetic field applied close to the hard axis is defined not only by the longitudinal magnetization component $M_{x}$, but also by the transverse $M_{y}$ component through the terms quadratic in magnetization $\left(\sim M_{x} M_{y}\right.$ type) in the tensor of the dielectric susceptibility. ${ }^{9,31-33}$ This leads to the magnetic field asymmetry of the measured field dependencies, and the observation that they not exactly reflect the behavior of the $M_{x}(H)$ component. The separation of contributions linear and quadratic in magnetic field can be performed with the help of a loop symmetrization procedure. The dependencies shown in Fig. 6 are the result of such a procedure and reflect the behavior of the longitudinal $M_{x}$ component. As follows from the Fig. 6, the MOKE weakly depends on $\xi$ at small $\xi$ values. That is, because the longitudinal MOKE is sensitive to the variation of the $M_{x}$ component and not sensitive to the formation of a domain structure with different orientations of $M_{y}$ components in the domains. After removing the magnetic field the magnetization in the domains is oriented along the easy axes $\left(\mathbf{e}_{1}\right)$ and $\left(\mathbf{e}_{2}\right)$, with the $M_{x}$ components being equal, but $M_{y}$ differing in sign at $\xi=0$. Therefore, the MOKE field dependence corresponding to a multidomain state at $H=0$ [Fig. $6(\mathrm{~b})]$ is similar to that realized for the monodomain state [Figs. 6(a) and 6(c)].

\section{Magnetic anisotropy fluctuations}

It is known that for large values of the demagnetizing field the magnetization in $\mathrm{Fe} / \mathrm{Cr}$ and $\mathrm{Fe} / \mathrm{Cr} / \mathrm{Fe}$ structures lies in the film plane. Such a situation is realized for film thicknesses larger than $d=8-10 \mathrm{ML}$, if the perpendicular interfacial anisotropy energy is smaller than the volume one, i.e., its magnitude is insufficient to turn the magnetization out of plane. $^{6,34,35}$ In this case the in-plane magnetization orientation at $H=0$ is defined by the in-plane magnetic anisotropy energy and its angular dependence. The Fe films grown on $\mathrm{GaAs}(100)$ substrates with $\mathrm{Ag}$ as a buffer layer display a fourfold (biaxial) in-plane magnetic anisotropy with the directions of two easy $\left(\mathbf{e}_{1}\right.$ and $\left.\mathbf{e}_{2}\right)$ and two hard $\left(\mathbf{h}_{1}\right.$ and $\left.\mathbf{h}_{2}\right)$ axes along [100]- and [110]-type crystallographic directions, respectively. ${ }^{36-38}$

In principle, in the absence of an external magnetic field the magnetization $\mathbf{M}$ might be directed along two mutually perpendicular directions $\mathbf{e}_{1}$ or $\mathbf{e}_{2}$ in the film plane, thereby forming a domain structure with four possible orientations of the magnetization vector in the domains. However, in an idealized perfect film, i.e., infinite extension in the film plane, such a structure is not favorable, because of the additional energy needed to nucleate domain walls. It should be noted, however, that in contrast to the bulk samples where the domain formation results in energy gain due to the compensation of demagnetizing fields, there is no energy gain due to domain formation in thin films, since the demagnetizing fields in the film plane are equal to zero (this holds, of course, only if the influence of the boundary can be neglected-otherwise there will be closure domains formed). Therefore, it is believed ${ }^{35,39,40}$ that after the external magnetic field is reduced to zero a monodomain state develops, with the direction of the magnetization pointing along one of the easy axes, namely, the one that is closer to the direction of magnetic field applied earlier.

However, if the magnetic field is applied exactly along a hard axis $(\mathbf{H} \| \mathbf{h})$, there are two equivalent easy axes $\left(\mathbf{e}_{1}\right.$ and $\mathbf{e}_{2}$ ) oriented at angles of $45^{\circ}$ to the direction of the magnetic field $(\mathbf{H})$ and the hard axis $(\mathbf{h})$. This results in a situation 
known as "Buridan's donkey": the magnetization has no way to decide which direction is more favorable to rotate into as the magnetic field slowly decreases from the saturation value $\left(H=H_{s}\right)$ to zero. Therefore, the question arises: In what manner will the magnetization reversal take place in this particular case?

Two extreme variants of the magnetization reversal process can be considered, related to either an ideal or a more realistic picture of the thin film. (i) Even infinitely small deflections of the magnetic field from the hard axis $\mathbf{h}$ result in a symmetry breaking and thus a nonequivalency of the easy axes. The magnetization will then coherently rotate toward the nearest easy axis without a domain structure formation as the field decreases. This seems to be the energetically most favorable variant, because the energy losses associated with the formation of domain walls are absent in this case. In practice, the realization of the situation $\mathbf{H} \| \mathbf{h}$ is extremely difficult to achieve, because there may be always an arbitrarily small field component perpendicular to $\mathbf{h}$, leading to a symmetry breaking. (ii) The uncertainty of the magnetization rotation might be removed by assuming that the easy axes $\left(\mathbf{e}_{1}\right.$ and $\mathbf{e}_{2}$ ) are varying across the film surface, i.e., locally the easy axis fluctuates around the average direction of $\mathbf{e}_{1}$ or $\mathbf{e}_{2}$. This fluctuation of the easy axis direction, which more closely describes the situation in a realistic film, in turn, may be caused by fluctuations of the magnetic anisotropy energy $\delta \varepsilon_{m}(\mathbf{r})$ due to defects or strains, or to a locally varying exchange coupling between $\mathrm{Fe}$ and $\mathrm{Cr}$ magnetic moments at the $\mathrm{Fe} / \mathrm{Cr}$ interface. As a result, in different film areas the preferable direction of the magnetization rotation will correspond to differently oriented easy axes $\mathbf{e}_{1}$ and $\mathbf{e}_{2}$. This situation is a precondition for the formation of domain structure at $H=0$ after magnetizing along the hard axis.

In strong magnetic fields the Zeeman energy essentially overcomes the magnetic anisotropy energy and the magnetization aligns with the field, i.e., $\mathbf{M} \| \mathbf{H}$. As the magnetic field decreases, the influence of the magnetic anisotropy becomes stronger and the magnetization tends to rotate toward the nearest easy axis. If in different film areas the directions of the easy axes are fluctuating, the formation of nonuniform distribution of the magnetizations in domains occurs. The exchange coupling acts against this process, because it favors parallel alignment of the magnetization in different areas. However, the energy losses required for the formation of a nonuniform structure are small, at least in relatively large fields and can be compensated by a gain in magnetic anisotropy energy. It seems that this situation is somewhat analogous to the one considered in the "random anisotropy" model, ${ }^{41}$ used for the description of magnetic properties of amorphous alloys or polycrystalline magnetics. The experiment shows that this nonuniform magnetization distribution is kept down to $H=0$, where a multidomain state with $90^{\circ}$ domains is realized.

The magnetic energy with lateral fluctuations taken into account can then be written in the form

$$
\varepsilon_{m}(\mathbf{r})=-H M_{s} \cos \psi+\frac{K_{1}}{4} \cos ^{2}(2 \varphi)+\delta \varepsilon_{m}(\mathbf{r})
$$

The fluctuating part $\delta \varepsilon_{m}(\mathbf{r})$ that provides the local nonequivalency of the easy axes can be written as follows:

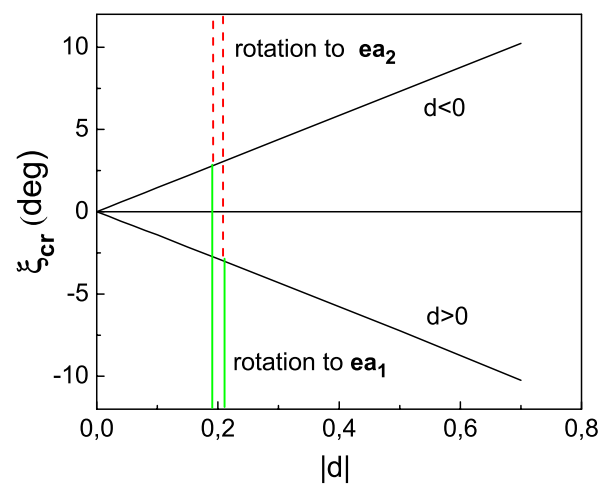

FIG. 7. (Color online) Calculated dependencies of the critical azimuth of the hard axis $\xi_{\mathrm{cr}}$ as a function of $|d|=\left|2 b / K_{1}\right|$ for opposite signs of $d$.

$$
\delta \varepsilon_{m}(\mathbf{r})=b(\mathbf{r})(\mathbf{u} \cdot \mathbf{m})^{2}
$$

where the coordinate function $b(\mathbf{r})$ might have a different sign in different areas of the film; $\mathbf{m}$ is the unit vector along the magnetization direction; $\mathbf{u}$ is the unit vector along one of the easy axes, e.g., $\mathbf{e}_{1}$. The addition of the fluctuating uniaxial anisotropy to $\varepsilon_{m}$ results in a situation in which one of the easy axes locally becomes an intermediate axis, while the second easy axis is the only remaining one. For example, assuming that $|b|<\left|K_{1}\right|$, at $b<0(b>0)$ the easy axis will be $\mathbf{e}_{1}\left(\mathbf{e}_{2}\right)$ and the middle axis will be $\mathbf{e}_{2}\left(\mathbf{e}_{1}\right)$. If the magnetic field is applied along the hard axis, $\mathbf{H} \| \mathbf{h}_{1}$, then as the magnetic field decreases from $H_{s}$ to zero in the areas where $b$ $<0$, the magnetization will tend to rotate toward $\mathbf{e}_{1}$, while in the $b>0$ areas to the $\mathbf{e}_{2}$ axis. It is important to note that, for $b<0(b>0)$, the magnetization will rotate toward $\mathbf{e}_{1}\left(\mathbf{e}_{2}\right)$ not only at $\mathbf{H} \| \mathbf{h}_{1}$, but also in the case when the direction of $\mathbf{h}_{1}$ slightly deviates from the field direction, so that the middle axis $\mathbf{e}_{2}\left(\mathbf{e}_{1}\right)$ lies closer to the field direction than to the easy axis. The maximum value of this deflection (the critical azimuth of the hard axis $\xi_{\mathrm{cr}}$ ) depends on the sign and value of the parameter $d=2 b / K_{1}$. The calculated dependencies of the critical azimuthal angle $\xi_{\text {cr }}$ on $d$ for positive and negative values of $d$ are presented in Fig. 7. In the calculations it was assumed that $b(\mathbf{r})=$ const, i.e., we assume that in the area of each domain the function $b(\mathbf{r})$ might be replaced by its average (effective) value.

In particular, for $d=+0.2(b>0)$ as the field decreases from $+H_{s}$ to zero, the magnetization will rotate to $\mathbf{e}_{2}$ until the azimuth of the magnetic field decreases down to $-2.9^{\circ}$ (dashed red line approaching from the top the line with $d$ $>0$ ). At smaller azimuthal angles of the magnetic field the rotation will happen in the other direction (green line). At $d=-0.2(b<0)$ the magnetization will rotate to $\mathbf{e}_{1}$ until the azimuth of the magnetic field reaches $2.9^{\circ}$ (green line approaching from the bottom the line $d<0$ ). At larger azimuth values the magnetization rotates to $\mathbf{e}_{2}$ (dashed red line). As seen in Fig. 7, for $|d| \neq 0$ there is always a hard axis azimuthal region, where for positive and negative values of $d$ a magnetization rotation happens in opposite directions as the field decreases. For $|d|=0.2$ that region is $-2.9^{\circ}<\xi<2.9^{\circ}$. At $\xi>2.9^{\circ}$ and $\xi<-2.9^{\circ}$ the rotation in all film areas will 
happen in one direction (toward $\mathbf{e}_{2}$ or $\mathbf{e}_{1}$, respectively). As follows from the experiment (see Fig. 5) for the film with a thickness of $49 \mathrm{~nm}$, the critical angle has the value about $\xi_{\mathrm{cr}} \approx 0.3^{\circ}$, since for $|\xi|>0.3^{\circ}$ the field dependencies $I_{2 \omega}(H)$ are well described by a coherent magnetization rotation for both positive and negative fields. For $|\xi|<0.3^{\circ}$ the multidomain magnetic structure is observed in the film. That allows one to estimate the average value of the parameter $d$, which is $d \approx 0.01$. From that it follows that even small $\sim 1 \%$ fluctuations of the magnetic anisotropy energy might result in essential changes of the magnetic structure in biaxially anisotropic films. These changes are manifested at a magnetic field direction near the hard axis when there are two equivalent magnetic states differing only in the sign of the $M_{y}$ component.

The fluctuations of the magnetic anisotropy energy might arise for different reasons. (i) It is known that at the $\mathrm{Fe} / \mathrm{Ag}$ or $\mathrm{Fe} / \mathrm{Cr}$ interfaces strained regions may form, because of the difference in unit cell parameters or due to interfacial alloying of $\mathrm{Cr}$ and $\mathrm{Fe}$ ions. ${ }^{6}$ The fluctuations of strain values in the film might result in lateral fluctuations of the magnetic anisotropy energy $\delta \varepsilon_{m}(\mathbf{r})$ described by Eq. (7). Analogous fluctuations of $\delta \varepsilon_{m}(\mathbf{r})$ can arise due to the existence of atomic scale steps and terraces, islands, and pits at the $\mathrm{Ag} / \mathrm{Fe}$ and $\mathrm{Fe} / \mathrm{Cr}$ interfaces oriented along fourfold axes. ${ }^{42}$ (ii) The fluctuations $\delta \varepsilon_{m}(\mathbf{r})$ may arise as a consequence of the antiferromagnetic exchange interaction between the interfacial magnetizations of $\mathrm{Fe}$ and $\mathrm{Cr}$. It is known that in thin $\mathrm{Cr}$ films at $T<T_{N}$ there is a layer-by-layer antiferromagnetic ordering of the magnetic moments. ${ }^{5}$ In addition, in the $\mathrm{Fe} / \mathrm{Cr}$ interface areas with enhanced concentration of atomic scale roughnesses biquadratic coupling might take place even in the case of one interface. ${ }^{22}$ If the antiferromagnetic state of the film is monodomain, then in the areas with a large amount of roughness the magnetization direction, which is orthogonal to that occurring in the areas with lower roughness, is more profitable. If in the $\mathrm{Cr}$ film the antiferromagnetic multidomain structure with orientation of the antiferromagnetic vector $\mathbf{L}$ along directions of [001] type is realized, then, even in the case of a uniform roughness distribution, the preferable magnetization direction is [010] in the areas with $\mathbf{L} \|[001]$, and

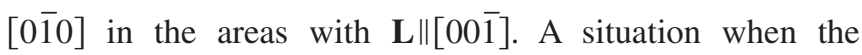
changes happen in magnetic structures of both $\mathrm{Fe}$ and $\mathrm{Cr}$ upon magnetizing is also possible. Clearly, separation of the mechanisms of (i) and (ii) should be possible by means of temperature-dependent studies. At a temperature remarkably higher than $T_{N}\left(T>1.8 T_{N}\right.$; Ref. 43$)$ where $\mathrm{Cr}$ is in the paramagnetic state, the mechanism (ii) should disappear. The contribution resulting from the mechanism (i) may change with temperature, but should survive above $T_{N}$.

\section{CONCLUSIONS}

Thus, the investigations of monocrystalline $\mathrm{Fe} / \mathrm{Cr}$ films with biaxial magnetic anisotropy show that, when the magnetic field is close to the hard axis direction, the remagnetization process is accompanied by multidomain structure formation. The multidomain structure appears not only in the narrow field region corresponding to the domain wall movement occurring during remagnetization, but in a much wider field range including also $H=0$. The domains formed have equal magnetization components $\left(M_{x}\right)$ along the magnetic field, but magnetization components $\left(M_{y}\right)$ perpendicular to $\mathbf{H}$ of opposite signs. It is shown that in the situation with equal domain volumes having opposite directions of $M_{y}$ components and, as a result, with zero sum, a transverse magnetization can be realized at orientations of the magnetic field close to the hard axis. The reason for the multidomain structure formation is proposed to lie in lateral fluctuations of the magnetic anisotropy energy arising in the film because of defects or strains or due to the presence of atomic scale roughness at the interface. The existence of multidomain structure can manifest itself in magnetization dynamics experiments and should be taken into account for the interpretation of experimental results (BLS, FMR).

\section{ACKNOWLEDGMENTS}

The authors would like to thank R. Schreiber for the sample preparation. For A.A.R. and B.B.K. this work was supported by RFFI Project No. N 05-02-16451-a.
${ }^{1}$ M. N. Baibich, J. M. Broto, A. Fert, F. Nguyen Van Dau, F. Petroff, P. Etienne, G. Creuzet, A. Friederich, and J. Chazelas, Phys. Rev. Lett. 61, 2472 (1988).

${ }^{2}$ G. Binasch, P. Grünberg, F. Saurenbach, and W. Zinn, Phys. Rev. B 39, 4828 (1989).

${ }^{3}$ S. A. Wolf, D. D. Awschalom, R. A. Buhrman, J. M. Daughton, S. von Molnar, M. L. Roukes, A. Y. Chtchelkanova, and D. M. Treger, Science 294, 1488 (2001).

${ }^{4}$ G. A. Prinz, Science 282, 1660 (1998).

${ }^{5}$ H. Zabel, J. Phys.: Condens. Matter 11, 9303 (1999).

${ }^{6}$ B. Heinrich and J. F. Cochran, Adv. Phys. 42, 523 (1993).

${ }^{7}$ S. O. Demokritov, A. B. Drovosekov, N. M. Kreines, H. Nembach, M. Rickart, and D. I. Kholin, JETP 95, 1062 (2002).

${ }^{8}$ D. E. Bürgler, P. Grünberg, S. O. Demokritov, and M. T.
Johnson, in Handbook of Magnetic Materials, edited by K. H. J. Buschow (Elsevier Science, Amsterdam, 2001), Vol. 13.

${ }^{9}$ A. A. Rzhevsky, B. B. Krichevtsov, D. E. Bürgler, and C. M. Schneider, Phys. Rev. B 75, 144416 (2007).

${ }^{10}$ M. Fiebig, D. Frohlich, St. Leute, and R. V. Pisarev, Appl. Phys. B: Lasers Opt. 66, 265 (1998)

${ }^{11}$ M. Fiebig, D. Fröhlich, K. Kohn, St. Leute, Th. Lottermoser, V. V. Pavlov, and R. V. Pisarev, Phys. Rev. Lett. 84, 5620 (2000).

${ }^{12}$ M. Fiebig, Th. Lottermoser, D. Frohlich, A. V. Goltsev, and R. V. Pisarev, Nature (London) 419, 818 (2002).

${ }^{13}$ A. Kirilyuk and Th. Rasing, J. Opt. Soc. Am. B 22, 148 (2005).

${ }^{14}$ V. Kirilyuk, A. Kirilyuk, and Th. Rasing, Appl. Phys. Lett. 70, 2306 (1997).

${ }^{15}$ I. L. Lyubchanskii, A. V. Petukhov, and Th. Rasing, J. Appl. 
Phys. 81, 5668 (1997).

${ }^{16}$ N. N. Dadoenkova, I. L. Lyubchanskii, M. I. Lyubchanskii, and Th. Rasing, Appl. Phys. Lett. 74, 1880 (1999).

${ }^{17}$ J. Hamrle, L. Poloreckŷ, and J. Ferré, Phys. Rev. B 68, 144401 (2003).

${ }^{18}$ R. F. Soohoo, Magnetic Thin Films (Harper \& Row, New York, 1965), p. 188.

${ }^{19}$ A. Hubert and R. Schäfer, Magnetic Domains: The Analysis of Magnetic Microstructures (Springer-Verlag, Berlin, 1998), p. 696.

${ }^{20}$ A. Stankiewitcz, A. Maziewski, J. Ferré, and V. Grolier, J. Appl. Phys. 74, 2519 (1999)

${ }^{21}$ P. Fischer, T. Eimüller, G. Schütz, M. Köhler, G. Bayreuther, G. Denbeaux, and D. Attwood, J. Appl. Phys. 89, 7159 (2001).

${ }^{22}$ H. Hopster, Phys. Rev. Lett. 83, 1227 (1999); J. Appl. Phys. 87, 5475 (2000).

${ }^{23}$ C. M. Schneider, K. Meinel, J. Kirschner, M. Neuber, C. Wilde, M. Grunze, K. Holldack, Z. Celinski, and F. Baudelet, J. Magn. Magn. Mater. 162, 7 (1996).

${ }^{24}$ D. E. Bürgler, C. M. Schmidt, D. M. Schaller, F. Meisinger, R. Hofer, and H.-J. Güntherodt, Phys. Rev. B 56, 4149 (1997).

${ }^{25}$ K. H. Bennemann, J. Magn. Magn. Mater. 200, 679 (1999).

${ }^{26}$ W. Hübner and K. H. Bennemann, Phys. Rev. B 52, 13411 (1995).

${ }^{27}$ L. C. Sampaio, J. Hamrle, V. V. Pavlov, J. Ferré, P. Georges, A. Brun, H. Le Gall, and J. Ben Youssef, J. Opt. Soc. Am. B 22, 119 (2005).

${ }^{28}$ R. Atkinson and N. F. Kubrakov, Phys. Rev. B 65, 014432 (2001); Appl. Phys. B: Lasers Opt. 74, 697 (2002).

${ }^{29}$ A. K. Zvezdin and V. A. Kotov, Modern Magnetooptics and Magnetooptical Materials (Institute of Physics Publishing, Bris- tol, 1997).

${ }^{30}$ L. C. Sampaio, J. Hamrle, A. Mougin, J. Ferré, F. Garcia, F. Fettar, B. Dieny, and A. Brun, Phys. Rev. B 70, 104403 (2004).

${ }^{31}$ R. M. Osgood III, B. M. Clemens, and R. L. White, Phys. Rev. B 55, 8990 (1997).

${ }^{32}$ A. A. Rzhevsky, B. B. Krichevtsov, D. E. Bürgler, and C. M. Schneider, Phys. Rev. B 75, 224434 (2007).

${ }^{33}$ B. B. Krichevtsov, A. K. Kaveev, A. Balanev, N. S. Sokolov, J. Camarero, and R. Miranda, Phys. Solid State 49, 1481 (2007).

${ }^{34}$ A. Murayama, K. Hyomi, J. Eickmann, and C. M. Falko, J. Magn. Magn. Mater. 198-199, 372 (1999).

${ }^{35}$ M. G. Pini, P. Politi, A. Rettori, G. Carlotti, G. Gubbiotti, M. Madami, and S. Tacchi, Phys. Rev. B 70, 094422 (2004).

${ }^{36}$ B. Heinrich, J. F. Cochran, A. S. Arrott, S. T. Purcell, K. B. Urquhart, J. R. Dutcher, and W. F. Egelhoff, Jr., Appl. Phys. A: Solids Surf. 49, 473 (1989).

${ }^{37}$ R. J. Hicken, A. Ercole, S. J. Gray, C. Daboo, and J. A. C. Bland, J. Appl. Phys. 79, 4987 (1996).

${ }^{38}$ R. J. Hicken, S. J. Gray, A. Ercole, C. Daboo, D. J. Freeland, E. Gu, E. Ahmad, and J. A. C. Bland, Phys. Rev. B 55, 5898 (1997).

${ }^{39}$ P. Politi and M. G. Pini, Eur. Phys. J. B 2, 475 (1998).

${ }^{40}$ C. Stamm, F. Marty, A. Vaterlaus, V. Weich, S. Egger, U. Maier, U. Ramsperger, H. Fuhrmann, and D. Pescia, Science 282, 449 (1998).

${ }^{41}$ P. Alben, J. J. Becker, and M. C. Chi, J. Appl. Phys. 49, 1653 (1978)

${ }^{42}$ R. A. Hyman, A. Zangwill, and M. D. Stiles, Phys. Rev. B 60, 14830 (1999).

${ }^{43}$ D. T. Pierce, R. J. Celotta, and J. Unguris, J. Appl. Phys. 73, 6201 (1993). 